\section{Taxonomists are an endangered species in Europe}

Sir - Studying and making an inventory of the planet's vanishing biodiversity are apparently not of great concern to European decision-makers. Taxonomists face increasing difficulty in finding work and funding. Many European universities no longer offer taxonomy courses. It is getting harder to maintain important specimen collections and handle loans.

There has been a catastrophic reduction in cataloguing the still largely undescribed tropical biota - the West's responsibility, as there are few trained taxonomists in tropical countries. Compiling an inventory of the tens of thousands of larger fungi in tropical Africa depends on three or four European mycologists. And this at a time when the scientific community has started to realize how much fungal diversity has been underestimated in the past - barely $10 \%$ of organisms have been described.

Several French and Dutch research centres with international reputations in taxonomic mycology have been dramatically cut in size. In Britain, retiring taxonomists at the botanic gardens of Kew and Edinburgh have not been replaced, and the International Mycological Institute has ceased its independent existence. As far as the taxonomy of most of the typical larger mushrooms is concerned, there is only one professional mycologist left in the Netherlands, two in France, and none in Britain. Switzerland looks likely to have none soon if Zurich follows the example of the University of Lausanne and fails to replace departing staff. Retiring mycologists are often heads of laboratories or research units, and their retirement usually puts an end to taxonomic mycology there.

In contrast, US institutions such as the Missouri Botanical Garden are dynamic and growing fast. With a policy of permanent presence on all continents and scientists at Europe's most famous institutions, US efficiency is imposing itself on one of the richest legacies of European history. Bart Buyck

Muséum National d'Histoire Naturelle, Laboratoire de Cryptogamie, 12 rue Buffon, 75005 Paris, France

\section{Don't leave the biology out of bioinformatics}

Sir - Marlie MacLean and Colin Miles highlight the skills gap for 'bioinformaticians' in the United Kingdom and elsewhere (Nature 401, 10; 1999). There is little doubt that, given the resources, it will be possible to find many people capable of manipulating the enormous volumes of data being generated, but such people must have a knowledge of and genuine interest in biology. The solution is either to identify those who are adept at both computer science and biology, which will be difficult, or to create interdisciplinary teams, which is a much more realistic approach.

The present headlong rush to expand bioinformatics could lead to the creation of isolated groups that have little interaction with molecular biologists and biochemists. If this occurs, the subject will fail to achieve the promise that we are led to expect in terms of a better understanding of biology and disease.

Peter Campbell

Department of Biochemistry and Molecular Biology, University College London, London WC1E 6BT, UK

\section{Courage could win back confidence in science}

Sir - Bradford Hawkins recently described an increase in the number of technical and trivial errors appearing in the published scientific literature (Nature $\mathbf{4 0 0}, 498 ; 1999)$. This, it was proposed, is a consequence of the 'publish or perish' philosophy of academic institutions. I would like to draw attention to an equally destructive trend: that of publishing banal descriptive reports that fail to enthuse the reader, or indeed the author.

The educational path to becoming a research scientist teaches the importance of detail, experimental rigour and assimilation of large amounts of scientific fact. New graduates are efficient at compiling literature searches, conducting experiments and analysing data. They are often eager to ask and answer fundamental questions of science and fit their experimental findings into a wider perspective of principle. However, they soon find that the scientific community does not reward them for this approach. Until recently, funding bodies and scientific publishers (presumably on advice from their reviewers) have executed a precautionary principle, favouring a conservative approach in scientific proposal and interpretation. The result is a failure of confidence to hypothesize.

The need for imaginative science is at last being recognized by major funding bodies - the W. M. Keck Foundation, among others - that provide funding for young investigators to establish more creative research projects. But, so far, this philosophy is rarely supported in the publication of scientific reports. It is more difficult to get a speculative comment through a reviewer than it is to publish several megabases of genetic sequence. Max Perutz recently drew attention to the plight of too many young postdocs with diminishing career prospects
(Nature 399, 299-301; 1999). This indirectly encourages predictable research and publication of trite interpretations of data instead of new insights, because, understandably, scientists are more interested in remaining employed than in taking the risk of rejection from reviewers.

There is an urgent need for the responsible communication of scientific discovery to counteract the public's loss of faith in science. While being didactic in form, it should convey a sense of the excitement and momentum of scientific discovery without being sensationalist. What better place to start than in the scientific press? For this to happen, publishers, reviewers and scientists need encouragement to be brave. Susan Daenke

Nuffield Department of Clinical Medicine, University of Oxford, John Radcliffe Hospital, Oxford OX3 9DU, UK

\section{Sprucing up one's impact factor}

Sir-Journal impact factors (IFs) were introduced in the 1970s to rank different journals by citation analysis ${ }^{1}$. IF is defined as the mean number of citations received in a particular year to articles published in the journal in the preceding two years.

It has been recognized for some time that the absolute values of IF, for a given qualitative journal standard, vary widely among disciplines (for example, biology, chemistry and engineering) and that review (secondary publication) journals have much higher IFs than do the primary publication journals carrying original research articles. Nevertheless, the IF has become an important parameter helping librarians to make difficult decisions concerning journal subscriptions, and consequently for the journals themselves in their attempts to increase sales and advertising revenues.

We wished to determine recent changes in the editorial or publication policies of different journals in biomedical fields that may have a bearing on their IFs. Our search has been limited, but the following findings are noteworthy.

(1) Since the early 1990s, many primarypublication biology journals have introduced 'mini-reviews' or their equivalent. Review articles attract citations more rapidly, and in larger numbers, than primary articles.

(2) Research articles in medical journals are often the subject of short comments that are published, with a response from the original authors, in a subsequent issue. For the first time in its 175-year history, The Lancetaltered its policy in 1996 so that an explicit (that is, countable) citation to the original article is now included in every one 
of the published letters of comment; earlier, such citations were included in the text itself and were not countable.

(3) Perhaps most important, one must consider the case of articles that are not counted as 'source' items (for the denominator) in the calculation of IF. These include editorials, book reviews, letters to the editor, and obituaries. Perhaps surprisingly, citations to such 'non-source' articles are counted in the numerator in the IF calculation. For the journals concerned, such items may therefore be taken to represent instances of 'nothing lost, anything gained'. Included in the examples of non-source articles are the numerous short clinical or laboratory study reports published as Letters to the Editor in The Lancet, some of which may even be considered as citation classics; a pair ${ }^{2,3}$ in 1993 on non-01 cholera received approximately 200 citations in the next two years.

Some categories of published items originally classified as source articles have been reclassified as non-source. For example, meeting abstracts published in FASEB Journal were reclassified as non-source articles from 1988, and the IF for the journal registered a leap from 0.24 in 1988 to 18.3 in 1989. In 1983, Nature started publishing Scientific Correspondence which, together with the prestigious News and Views section, now comprises a large repository of citable non-source articles. Many other journals appear to be following these trends. When one compares the number of pages devoted to source articles with those for non-source items, the ratio for Nature had more than halved, from 3.5 in January 1977 to 1.6 twenty years later, even though the total number of pages in the journal was virtually unchanged over this period. If nothing else, our findings support the case for a change in the present method of IF calculation, so that citations to source articles alone are counted.

Finally, we have identified a loophole that could allow a less than scrupulous, perhaps obscure, journal to increase its IF from 0.1 to a healthy 2.1 , by the mere expedient of adding two spurious self-citations in each of its source articles. Is it possible that this may be happening already?

\section{J. Gowrishankar, P. Divakar}

Centre for Cellular \& Molecular Biology,

Uppal Road, Hyderabad 500 007, India

Sir-Scientific institutions are increasingly judged on the quality of the journals in which their staff publish papers. Journal quality is usually presented as its impact factor (IF), the number of citations in a given year to papers published in the two previous years, divided by the number of items published in those previous two years (Journal Citation Reports, Institute for Scientific Information, 1997).
As veterinary researchers, we sometimes find ourselves searching for possible human angles in our work, so that we might publish in medical journals, which tend to have significantly higher IFs than their veterinary counterparts. But we have spotted a simpler and more effective approach that will allow us to publish in appropriate places and still get high ratings. As an example, The Veterinary Record has an IF of about 1 , based on approximately 600 citations and 600 papers published in 1995 and 1996. Our institute publishes about 300 papers in two years. Our director need only instruct us all to cite at least two papers from The Veterinary Record in every paper we publish from now on, however loose the connection, for the IF to quickly double. The Veterinary Record would move from being in the top $40 \%$ of journals to being in the top $15 \%$.

We could have an even greater impact on journals that publish fewer papers. For example, if our director applied this policy to Veterinary Research Communications, the IF of that journal would increase from less than 1 to more than 6 , moving it into the top $3 \%$ of journals. If our institute teamed up with two or three others, we could rapidly create a competitor to Nature. Unethical, perhaps, but legal and very much in our interest.

Matthew Baylis, Michael Gravenor,

\section{Rowland Kao}

Institute for Animal Health, Compton Laboratory, Compton, Newbury, Berkshire RG20 7NN, UK

1. Garfield, E. Science 178, 471-479 (1972).

2. Ramamurthy, T. et al. Lancet 341, 703-704 (1993).

3. Albert, T. J. et al. Lancet 341, 704 (1993).

\section{No dirty tricks in merger ballot}

Sir - Rex Dalton's article about the proposed merger of the Optical Society of America (OSA) and the International Society of Optical Engineers contains a number of misleading statements (Nature 400, 605; 1999).

Dalton refers to accusations that a recent membership mailing of OSA exemplified "inappropriate leadership tactics". In fact, this half-price membership effort is an annual drive that was offered to all nonmembers in the society's vast database. No one group or specific out-of-house list was targeted for this mailing, and the inference that this solicitation was made to influence the proposed merger vote is without merit.

Dalton's report of threatened legal action against an unnamed OSA scientist is false. A letter sent by OSA's executive director to the scientist clearly states concern over the unauthorized use of a mailing list, but it does not make a legal threat. The society has traditionally taken a respectful stance regarding paper and electronic-mail intrusion into the lives of our members and always has acted to protect its mailing lists as an asset of OSA.

The article also leaves the mistaken impression that opponents of the merger have somehow been silenced in this debate. The board of directors and the staff of OSA have worked diligently to ensure that accurate, timely and informative materials were (and are) provided to our members so that they can make an educated decision on the proposed unification. There have been open forums at society meetings, links provided to the opponents' website, allmember communications providing the opponents' views mailed at the society's expense, and a ballot package sent to all eligible voters presenting equally arguments for and against the proposal.

This vote is extremely important to the future of our society - and the field of optics - and every effort has been made to keep the information flow fair and balanced.

Elaine Gansz Bobo

Optical Society of America, 2010 Massachusetts Avenue NW, Washington DC 20036-1023, USA

\section{Call a halt to strong-arm tactics over GM crops}

Sir - It is surprising that representatives of an organization that is reported to use acts of destructive force to achieve social and political goals are given a forum in Nature on the subject of how to restore public trust in science. I refer to the Commentary by Greenpeace's Benny Haerlin and Doug Parr (Nature 400, 499; 1999). One such act is the destructive attack on fields of genetically modified crops by a group that included the head of Greenpeace UK, as reported by The New York Times (23 August).

Press accounts indicate that this was only the latest in a series of attacks by Greenpeace and allied organizations. How can we now tell if a future refusal by farmers to grow genetically modified crops will not really be based on fear disguised as conviction instead of genuine conviction?

European political and religious history is replete with groups that have found the use of apparently peaceful propaganda together with the selective use of brute force to be an effective tool to change public opinion. It is sad that this seems to be happening today in Britain.

Haerlin and Parr suggest that the values of society should be paramount in the debates they discuss. This would have been an interesting suggestion to give to Galileo. Is the use of force in civil discussions one of the values they have in mind?

Manfred Philipp

City University of New York, 250 Bedford Park Boulevard West, Bronx, New York 10468, USA 